\title{
Theoretical Analysis of Moving Reference Planes Associated with Unit Cells of Nonreciprocal Lossy Periodic Transmission-Line Structures
}

\author{
Suthasinee LAMULTREE ${ }^{1}$, Prayoot AKKARAEKTHALIN ${ }^{2}$, Danai TORRUNGRUENG ${ }^{3}$ \\ ${ }^{1}$ Dept. of Electronics and Telecommunication Engineering, Faculty of Engineering, Rajamangala \\ University of Technology Isan Khonkaen Campus, Khonkaen, 40000, Thailand \\ 2 Faculty of Engineering, King Mongkut's University of Technology North Bangkok, \\ Bangkok, 10800, Thailand \\ ${ }^{3}$ Dept. of Electrical and Electronic Engineering, Faculty of Engineering and Technology, \\ Asian University, Chon Buri, 20260, Thailand.
}

suthasinee.la@rmuti.ac.th, prayoot@kmutnb.ac.th, danait@asianu.ac.th

Submitted April 22, 2016 / Accepted October 3, 2016

\begin{abstract}
This paper presents a theoretical analysis of moving reference planes associated with unit cells of nonreciprocal lossy periodic transmission-line structures (NRLSPTLSS) by the equivalent bi-characteristic-impedance transmission line (BCITL) model. Applying the $B C I T L$ theory, only the equivalent BCITL parameters (characteristic impedances for waves propagating in forward and reverse directions and associated complex propagation constants) are of interest. An infinite NRLSPTLS is considered first by shifting a reference position of unit cells along TLs of interest. Then, a semi-infinite terminated NRLSPTLS is investigated in terms of associated load reflection coefficients. It is found that the equivalent BCITL characteristic impedances of the original and shifted unit cells are mathematically related by the bilinear transformation. In addition, the associated load reflection coefficients of both unit cells are mathematically related by the bilinear transformation. However, the equivalent BCITL complex propagation constants remain unchanged. $\mathrm{Nu}$ merical results are provided to show the validity of the proposed theoretical analysis.
\end{abstract}

\section{Keywords}

Unit cell, periodic transmission-line structure, bicharacteristic-impedance transmission line (BCITL), bilinear transformation

\section{Introduction}

Reciprocal and nonreciprocal periodic structures of transmission lines (TLs) have several practical applications in microwave technology; e.g., microwave filters, slow wave components, traveling-wave amplifiers, phase shift- ers and antennas [1-10]. In general, problems associated with these periodic structures have been analyzed based on the Floquet's theorem [5]. For nonreciprocal periodic structures, there are several papers discussing about the analysis and their useful applications in the literature [6-10]. Recently, the equivalent model based on bi-characteristic-impedance TLs (BCITLs) has been proposed to conveniently analyze terminated finite reciprocal lossy periodic TL structures [11]. However, only reciprocal BCITLs are presented in [11]. To extend the concept for a more general problem, nonreciprocal lossy periodic TL structures (NRLSPTLSs) are considered in this paper. In applying the BCITL model, only the equivalent quantities associated with each unit cell of NRLSPTLSs are employed; i.e., equivalent characteristic impedances and associated complex propagation constants for waves propagating in forward and reverse directions.

For the analysis of a unit cell of reciprocal periodic structures related to moving reference planes, published papers [12], [13] and book [5] discovered that the propagation wavenumber of the structures is not a function of the position of the reference plane, and associated characteristic impedances depend on the choice of the reference position of the unit cell. However, results in [12], [13] are derived based on Maxwell's equations via the scatteringmatrix equation and the assumption of reciprocity, which is unnecessarily complicated. In addition, the finding in [5] is valid for a specific case only. Furthermore, it is not obvious, for nonreciprocal periodic structures, how to define a unit cell properly for convenience in analysis due to associated nonreciprocity and possible asymmetry. Therefore, this paper aims to provide simple and generalized derivations for a nonreciprocal unit cell of NRLSPTLSs when a reference position of unit cells is shifted along TLs of interest, including the relationship between associated BCITL parameters of the original and shifted unit cells. 
This paper is organized as follows. Section 2 presents an analysis of infinite NRLSPTLSs based on the equivalent BCITL model. Semi-infinite NRLSPTLSs are analyzed in Sec. 3. An example of NRLSPTLSs is shown in Sec. 4 to show the validity of the proposed solutions. Finally, Section 5 provides conclusions.

\section{Analysis of Moving Reference Planes for Infinite NRLSPTLSs}

A finite NRLSPTLS of $M$ nonreciprocal lossy unit cells can be effectively modeled as a BCITL of length $M d$ as shown in Fig. 1, where $d$ is the length of each unit cell [14]. Note that $V_{m}$ and $I_{m}$ are the phasor voltage and the phasor current at the terminal of the $m^{\text {th }}$ unit cell (where $m=1,2, \ldots, M)$, respectively. Generally, nonreciprocal BCITLs possess the complex propagation constants $\gamma^{+}$and $\bar{\gamma}$ with corresponding complex characteristic impedances $Z_{0}^{+}$and $Z_{0}^{-}$for waves propagating in forward and reverse directions, respectively. In this section, an infinite NRLSPTLS is considered, which can be obtained from Fig. 1 by letting both ends approach infinity.

Using the transmission $(A B C D)$ matrix technique and eigenanalysis, it can be shown rigorously that $\gamma^{+}$and $\bar{\gamma}$ of nonreciprocal BCITLs possess two possible solutions each, as follows [14]:

$$
\begin{aligned}
& \gamma_{1,2}^{+}=\frac{1}{d} \ln \left[\frac{A+D \pm \sqrt{(A-D)^{2}+4 B C}}{2}\right], \\
& \gamma_{1,2}^{-}=-\frac{1}{d} \ln \left[\frac{A+D \pm \sqrt{(A-D)^{2}+4 B C}}{2}\right]
\end{aligned}
$$

where the subscript " 1,2 " indicates the choice of + and signs in the solutions, respectively. Note that the total $A B C D$ parameters of each nonreciprocal unit cell possess the property of $A D-B C \neq 1$ [1]. Since, $\gamma^{+}$and $\bar{\gamma}$ each possess two possible solutions, it seems that there are four possible combined solutions for waves propagating along the infinite NRLSPTLS for the (forward, reverse) wave. However, only two valid solutions $\left(\gamma_{1}^{+}, \gamma_{2}^{-}\right)$and $\left(\gamma_{2}^{+}, \gamma_{1}^{-}\right)$ must be chosen for the (forward, reverse) wave [14]. In addition, $Z_{0}^{ \pm}$of nonreciprocal BCITLs can be expressed in terms of the total $A B C D$ parameters of each nonreciprocal unit cell as follows [14]:

$$
\begin{aligned}
& Z_{0,(1,2)}^{+}=\frac{-2 B}{A-D \mp \sqrt{(A-D)^{2}+4 B C}}, \\
& Z_{0,(1,2)}^{-}=\frac{2 B}{A-D \mp \sqrt{(A-D)^{2}+4 B C}} .
\end{aligned}
$$

To be consistent with the two valid solutions for $\gamma^{+}$ and $\bar{\gamma}$ (i.e., $\left(\gamma_{1}^{+}, \gamma_{2}^{-}\right)$and $\left.\left(\gamma_{2}^{+}, \gamma_{1}^{-}\right)\right)$, only two corresponding

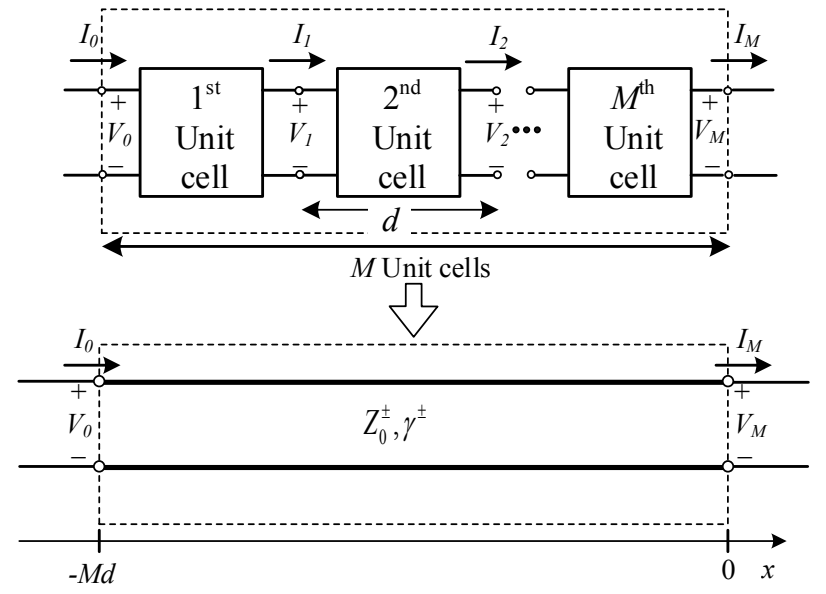

Fig. 1. A finite NRLSPTLS of $M$ unit cells and its equivalent BCITL model.

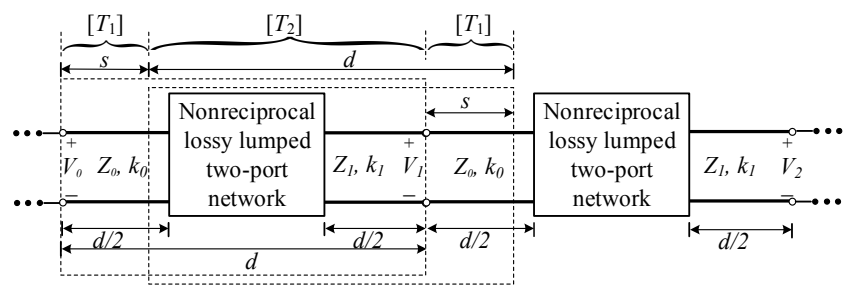

Fig. 2. A nonreciprocal unit cell of an infinite NRLSPTLS obtained by shifting a reference position $s$ along TLs.

solutions are valid; i.e., $\left(Z_{0,1}^{+}, Z_{0,2}^{-}\right)$and $\left(Z_{0,2}^{+}, Z_{0,1}^{-}\right)$. Thus, two sets of valid solutions of nonreciprocal BCITL parameters are given as follows:

(i) $\left(Z_{0,1}^{+}, \gamma_{1}^{+}\right)$and $\left(Z_{0,2}^{-}, \gamma_{2}^{-}\right)$for forward and reverse waves respectively,

(ii) $\left(Z_{0,2}^{+}, \gamma_{2}^{+}\right)$and $\left(Z_{0,1}^{-}, \gamma_{1}^{-}\right)$for forward and reverse waves, respectively.

To analyze NRLSPTLSs in the context of moving reference planes of unit cells, an original nonreciprocal unit cell of length $d$ is initially considered as shown in Fig. 2 (on the left end). In Fig. 2, an infinite NRLSPTLS consists of two distinct TLs, with the unloaded propagation constants $k_{0}$ and $k_{1}$ and the corresponding characteristic impedances $Z_{0}$ and $Z_{1}$, loaded with a nonreciprocal lossy lumped two-port network at the center. It should be pointed out that the two-port network is dimensionless. The reference position $(s)$ of the unit cell is shifted along TLs of interest, where $0 \leq s \leq d$. Since the structure of NRLSPTLS for both original and shifted cases are identical, $\gamma^{+}$and $\bar{\gamma}$ are expected to remain unchanged. To clarify this, let us consider Fig. 2, where the original unit cell can be considered as being composed of a cascade of two two-port networks possessing the transmission matrices $\left[\mathbf{T}_{1}\right]$ and $\left[\mathbf{T}_{2}\right]$. In addition, a shifted unit cell is composed of a cascade of two two-port networks possessing the transmission matrices $\left[\mathbf{T}_{2}\right]$ and $\left[\mathbf{T}_{1}\right]$, where

$$
\left[\mathbf{T}_{i}\right]=\left[\begin{array}{cc}
A_{i} & B_{i} \\
C_{i} & D_{i}
\end{array}\right],
$$


for $i=1$ and 2. In (5), $A_{i}, B_{i}, C_{i}$, and $D_{i}$ are the $A B C D$ parameters associated with $\left[\mathbf{T}_{i}\right]$. Note that the total transmission matrix of the original (when $s=0$ and $s=d$ ) and shifted unit cells can be written explicitly as (6) and (7), respectively:

$$
\begin{gathered}
{[\mathbf{T}]_{\mathrm{o}}=\left[\mathbf{T}_{1}\right]\left[\mathbf{T}_{2}\right]=\left[\begin{array}{ll}
A_{1} A_{2}+B_{1} C_{2} & A_{1} B_{2}+B_{1} D_{2} \\
C_{1} A_{2}+D_{1} C_{2} & C_{1} B_{2}+D_{1} D_{2}
\end{array}\right],} \\
{[\mathbf{T}]_{\mathrm{s}}=\left[\mathbf{T}_{2}\right]\left[\mathbf{T}_{1}\right]=\left[\begin{array}{ll}
A_{2} A_{1}+B_{2} C_{1} & A_{2} B_{1}+B_{2} D_{1} \\
C_{2} A_{1}+D_{2} C_{1} & C_{2} B_{1}+D_{2} D_{1}
\end{array}\right]}
\end{gathered}
$$

where the subscripts $o$ and $s$ in this paper are associated with the original and shifted unit cells, respectively. From (6) and (7), it can be shown that both cases provide the same $\gamma^{+}$and $\bar{\gamma}$, computed using (1) and (2), as

$$
\begin{aligned}
& \gamma_{1,2}^{+}=\frac{1}{d} \ln \left[\frac{W \pm \sqrt{X+Y}}{2}\right], \\
& \gamma_{1,2}^{-}=-\frac{1}{d} \ln \left[\frac{W \pm \sqrt{X+Y}}{2}\right],
\end{aligned}
$$

where

$$
\begin{aligned}
W & =A_{1} A_{2}+B_{1} C_{2}+C_{1} B_{2}+D_{1} D_{2}, \\
X & =\left(A_{1} A_{2}\right)^{2}+\left(B_{1} C_{2}\right)^{2}+\left(C_{1} B_{2}\right)^{2}+\left(D_{1} D_{2}\right)^{2}, \text { and } \\
Y & =2\left[A_{1} A_{2}\left(B_{1} C_{2}+C_{1} B_{2}-D_{1} D_{2}\right)-B_{1} C_{1} B_{2} C_{2}\right. \\
& \left.+D_{1} D_{2}\left(B_{1} C_{2}+C_{1} B_{2}\right)+2\left(A_{1} D_{1} B_{2} C_{2}+B_{1} C_{1} D_{2} A_{2}\right)\right] .
\end{aligned}
$$

However, it can be shown using (3), (4), (6) and (7) that the equivalent characteristic impedances of the original and shifted unit cells are different $\left(Z_{0,(1,2), o}^{ \pm} \neq Z_{0,(1,2), s}^{ \pm}\right)$depending on the reference position, where $Z_{0,(1,2), o}^{ \pm}$and $Z_{0,(1,2), s}^{ \pm}$are equivalent characteristic impedances of the original and shifted unit cells, respectively. In addition, it is found that $Z_{0,(1,2), o}^{+}$and $Z_{0,(1,2), s}^{+}$, as well as $Z_{0,(1,2), o}^{-}$and $Z_{0,(1,2), s}^{-}$are mathematically related by the following $b i$ linear transformation [15]:

$$
\begin{aligned}
& Z_{0,(1,2), s}^{+}=\frac{a Z_{0,(1,2), o}^{+}+b}{c Z_{0,(1,2), o}^{+}+d_{0}}, \\
& Z_{0,(1,2), s}^{-}=\frac{a_{1} Z_{0,(1,2), o}^{-}+b_{1}}{c_{1} Z_{0,(1,2), o}^{-}+d_{1}},
\end{aligned}
$$

with $a d_{0}-b c \neq 0$ and $a_{1} d_{1}-b_{1} c_{1} \neq 0$ in general, where $b=b_{1}=0$ and

$$
\begin{gathered}
a=-a_{1}=-B_{1} A_{2}-D_{1} B_{2}, \\
c=c_{1}=C_{1} B_{2}-B_{1} C_{2}, \\
d_{0}=-d_{1}=-A_{1} B_{2}-B_{1} D_{2} .
\end{gathered}
$$

In (10) and (11), it should be pointed out that the equivalent characteristic impedances of the original unit cell

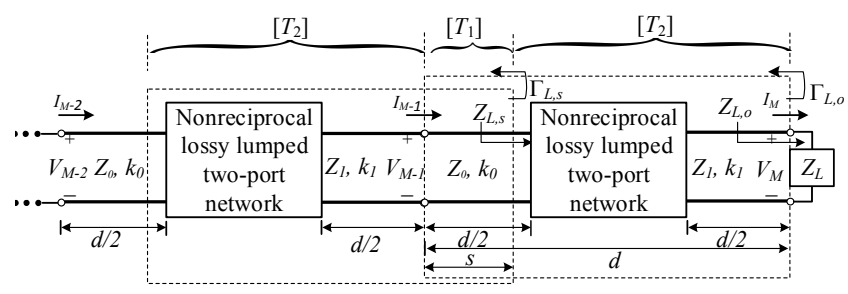

Fig. 3. A nonreciprocal unit cell of a semi-infinite NRLSPTLS terminated in a load impedance.

$Z_{0,(1,2), o}^{ \pm}$remain unchanged when moving reference planes due to the cascading property of the transmission matrix. Thus, once $Z_{0,(1,2), o}^{ \pm}$are known, only simple parameters in (12)-(14) are computed as moving reference planes. This is an advantages of using (10) and (11) in computing $Z_{0,(1,2), \mathrm{s}}^{ \pm}$ instead of (3) and (4) when studying effects of moving reference planes.

\section{Analysis of Moving Reference Planes for Semi-Infinite Terminated NRLSPTLSs}

Consider a semi-infinite NRLSPTLS, terminated in a load impedance $Z_{\mathrm{L}}$, as shown in Fig. 3, where $M$ approaches infinity. At the terminal of a nonreciprocal unit cell in Fig. 3, the load reflection coefficients of the original and shifted unit cells are mathematically defined in terms of associated load impedances as (15) and (16), respectively [14]:

$$
\begin{gathered}
\Gamma_{L,(1,2), o}=\frac{Z_{L, o} Z_{0,(2,1), o}^{-}-Z_{0,(1,2), o}^{+} Z_{0,(2,1), o}^{-}}{Z_{L, o} Z_{0,(1,2), o}^{+}+Z_{0,(1,2), o}^{+} Z_{0,(2,1), o}^{-}}, \\
\Gamma_{L,(1,2), s}=\frac{Z_{L, s} Z_{0,(2,1), s}^{-}-Z_{0,(1,2), s}^{+} Z_{0,(2,1), s}^{-}}{Z_{L, s} Z_{0,(1,2), s}^{+}+Z_{0,(1,2), s}^{+} Z_{0,(2,1), s}^{-}}
\end{gathered}
$$

where $Z_{\mathrm{L}, \mathrm{o}}$ and $Z_{\mathrm{L}, \mathrm{s}}$ are the load impedances seen at the end terminal of the original and shifted unit cells, respectively. In Fig. 3, $Z_{\mathrm{L}, \mathrm{o}}$ is equal to $Z_{\mathrm{L}}$. Using the transmission matrix technique, it can be shown that $Z_{\mathrm{L}, \mathrm{o}}$ and $Z_{\mathrm{L}, \mathrm{s}}$ are also mathematically related by the bilinear transformation as follows:

$$
Z_{L, s}=\frac{A_{2} Z_{L, o}+B_{2}}{C_{2} Z_{L, o}+D_{2}},
$$

with $A_{2} D_{2}-B_{2} C_{2} \neq 0$ in general. It can be shown that $\Gamma_{L,(1,2), o}$ and $\Gamma_{L,(1,2), s}$ are finally related by the bilinear transformation due to the bilinear transformation relationships between $\Gamma_{L,(1,2), o}$ and $Z_{\mathrm{L}, \mathrm{o}}, \Gamma_{L,(1,2), s}$ and $Z_{\mathrm{L}, \mathrm{s}}$, as well as $Z_{\mathrm{L}, \mathrm{o}}$ and $Z_{\mathrm{L}, \mathrm{s}}$, as follows:

$$
\Gamma_{L,(1,2), s}=\frac{a_{2} \Gamma_{L,(1,2), o}+b_{2}}{c_{2} \Gamma_{L,(1,2), o}+d_{2}},
$$

with $a_{2} d_{2}-b_{2} c_{2} \neq 0$ in general, where 


$$
\begin{aligned}
a_{2} & =Z_{0,(2,1), s}^{-}\left\{B_{2} Z_{0,(1,2), o}^{+}-A_{2} Z_{0,(1,2), o}^{+} Z_{0,(2,1), o}^{-}\right. \\
& \left.+Z_{0,(1,2), s}^{+} Z_{0,(2,1), s}^{-}\left(C_{2} Z_{0,(1,2), o}^{+} Z_{0,(2,1), o}^{-}-D_{2} Z_{0,(1,2), o}^{+}\right)\right\} \\
b_{2}= & -Z_{0,(2,1), s}^{-}\left\{B_{2} Z_{0,(2,1), o}^{-}+A_{2} Z_{0,(1,2), o}^{+} Z_{0,(2,1), o}^{-}\right. \\
& \left.+Z_{0,(1,2), s}^{+} Z_{0,(2,1), s}^{-}\left(C_{2} Z_{0,(1,2), o}^{+} Z_{0,(2,1), o}^{-}+D_{2} Z_{0,(2,1), o}^{-}\right)\right\}, \\
c_{2}= & Z_{0,(1,2), s}^{+}\left\{B_{2} Z_{0,(1,2), o}^{+}-A_{2} Z_{0,(1,2), o}^{+} Z_{0,(2,1), o}^{-}\right. \\
& \left.+Z_{0,(1,2), s}^{+} Z_{0,(2,1), s}^{-}\left(D_{2} Z_{0,(1,2), o}^{+}-C_{2} Z_{0,(1,2), o}^{+} Z_{0,(2,1), o}^{-}\right)\right\} \\
d_{2}= & -Z_{0,(1,2), s}^{+}\left\{B_{2} Z_{0,(2,1), o}^{-}+A_{2} Z_{0,(1,2), o}^{+} Z_{0,(2,1), o}^{-}\right. \\
& \left.-Z_{0,(2,1), s}^{-} Z_{0,(1,2), s}^{+}\left(C_{2} Z_{0,(1,2), o}^{+} Z_{0,(2,1), o}^{-}+D_{2} Z_{0,(2,1), o}^{-}\right)\right\}
\end{aligned}
$$

In (18), it should be pointed out that the load reflection coefficient of the original unit cell $\Gamma_{\mathrm{L},(1,2), \mathrm{o}}$ also remains unchanged when moving reference planes because $Z_{0,(1,2), \mathrm{o}}^{ \pm}$ and $Z_{\mathrm{L}, \mathrm{o}}$ in (15) remain unchanged as discussed at the end of Sec. 2 .

\section{An Example of NRLSPTLSs}

Consider an example of NRLSPTLSs implemented by a standard TL periodically loaded by a nonreciprocal microwave transistor as shown in Fig. 3. For this example, the bipolar junction transistor (BJT), Motorola MRF962 is selected to replace the nonreciprocal lossy lumped two-port network in Fig. 3. Only the operating frequency of $1.5 \mathrm{GHz}$ is considered in this paper, where the circuit parameters of the transistor are given as follows [16]: the collector-emitter voltage $V_{\mathrm{CE}}=10 \mathrm{~V}$, the collector current $I_{\mathrm{c}}=10 \mathrm{~mA}$ and the $S$ parameters in the $Z_{0}$ system with $S_{11}=0.77 \angle 168^{\circ}, \quad S_{12}=0.085 \angle 31^{\circ}, \quad S_{21}=1.72 \angle 55^{\circ}$ and $S_{22}=0.31 \angle-104^{\circ}$. The transistor is periodically loaded on the identical standard TL with the propagation constant $k$ $\left(k_{0}=k_{1}=k\right)$. It is assumed that the phase velocity of wave propagating along the standard TL is equal to $3 \times 10^{8} \mathrm{~m} / \mathrm{s}$. The length of TL $d$ of the unit cell is $6 \mathrm{~cm}$, and its characteristic impedance is $50 \Omega$. This NRLSPTLS is terminated in a $50 \Omega$-load impedance. In the example, it is assumed that each nonreciprocal unit cell is linear for the range of input voltages and currents of interest.

Using the standard formulas (3), (4) and the proposed bilinear-transformation formulas of (10)-(14), the equivalent BCITL characteristic impedances can be readily computed. Figure 4 shows the plot of the magnitude and the argument of $Z_{0,(1,2)}^{ \pm}$versus $s / d$. Note that $\phi_{1,2}^{+}$and $\phi_{1,2}$ are the arguments of $Z_{0,(1,2)}^{+}$and $Z_{0,(1,2)}$, respectively. It is found that $Z_{0,(1,2)}^{ \pm}$are different when varying $s / d$ as expected. The magnitudes of $Z_{0,(1,2)}^{ \pm}$can be varied significantly when moving reference planes as shown in Fig. 4(a) and (b). In addition, the arguments of $Z_{0,(1,2)}^{ \pm}$are different as expected since the considered unit cell is unsymmetrical as shown in Fig. 4(c) and (d). It is also observed that

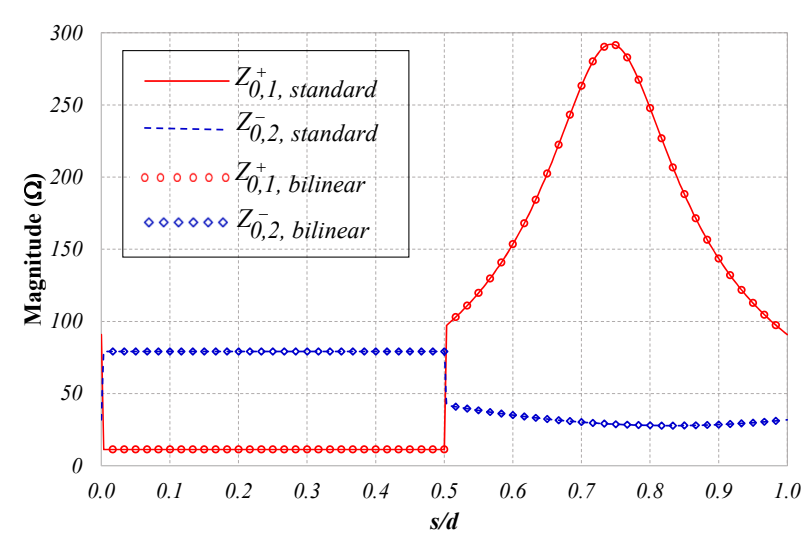

(a)

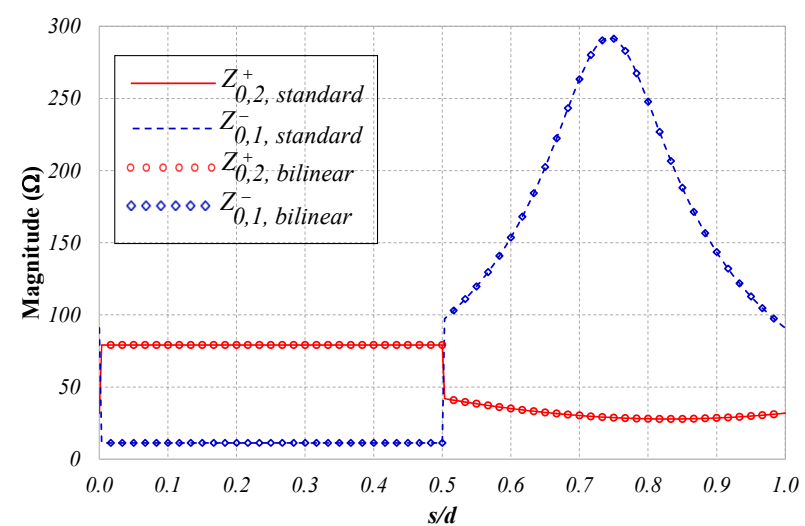

(b)

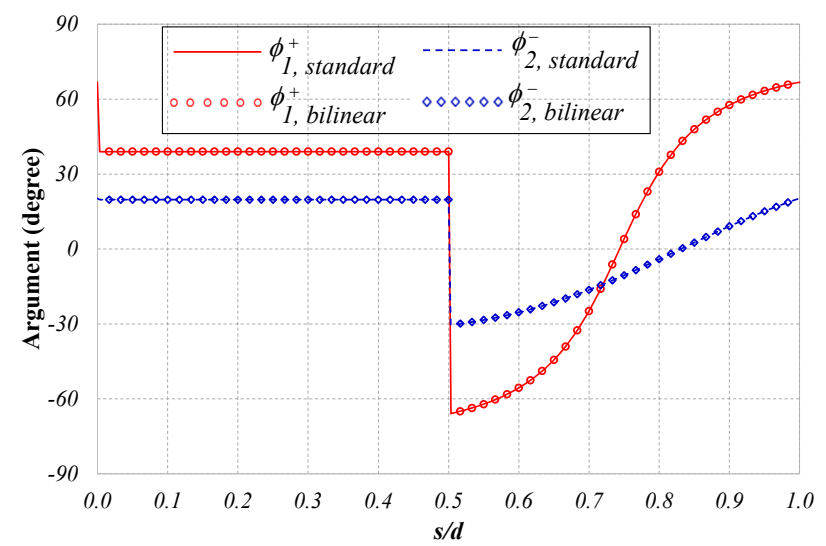

(c)

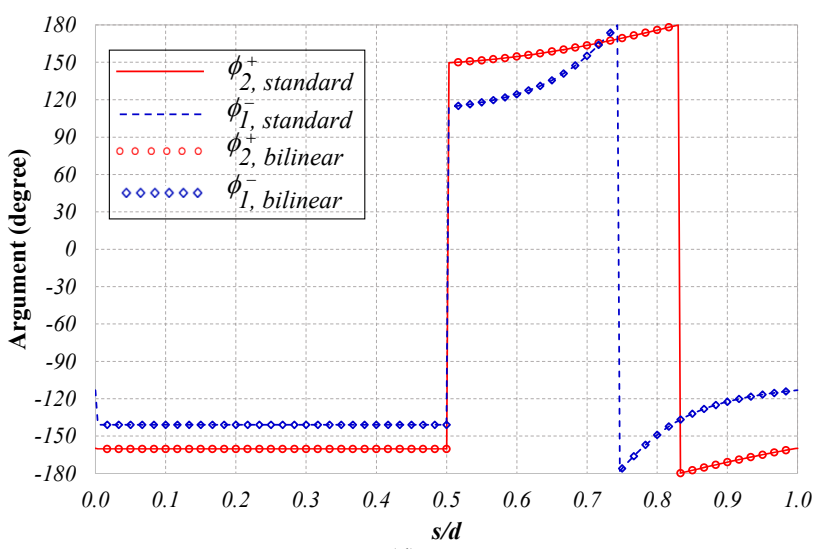

(d)

Fig.4. Equivalent BCITL characteristic impedances: (a) $\left|Z_{0,1}^{+}\right|$ and $\left|Z_{0,2}^{-}\right|$, (b) $\left|Z_{0,2}^{+}\right|$and $\left|Z_{0,1}^{-}\right|$, (c) $\phi_{1}^{+}$and $\phi_{2}^{-}$, (d) $\phi_{2}^{+}$and $\phi_{1}^{-}$. 
$Z_{0,(1,2)}^{ \pm}$are discontinuous at $s / d=0.5$ due to the presence of the transistor. When $s / d=0$ and $s / d=1$, all considered parameters are identical as expected because the shifted unit cell becomes the original unit cell again. Using either standard or proposed formulas, they provide the identical equivalent BCITL characteristic impedances. However, the proposed formulas provide more useful information of the relationship between equivalent BCITL characteristic impedances of the original and shifted unit cells.

Note that two sets of valid solutions of the equivalent BCITL complex propagation constants of unit cells of the example $\left(\left(\gamma_{1}^{+}, \gamma_{2}^{-}\right)\right.$and $\left.\left(\gamma_{2}^{+}, \gamma_{1}^{-}\right)\right)$remain unchanged as moving reference planes as expected, specifically $\gamma_{1}^{+}=(-5.94+\mathrm{j} 16.62) \mathrm{m}^{-1}, \quad \gamma_{2}^{-}=(44.19+\mathrm{j} 23.61) \mathrm{m}^{-1}$, $\gamma_{2}{ }^{+}=(-44.19-\mathrm{j} 23.61) \mathrm{m}^{-1}$, and $\gamma_{1}^{-}=(5.94-\mathrm{j} 16.62) \mathrm{m}^{-1}$.

Similarly, using the standard formula (16) and the proposed bilinear-transformation formulas of (18)-(22), the load reflection coefficient of the shifted unit cell can be readily computed. It is found that both approaches provide identical results, but the proposed formulas provide the relationship between the load reflection coefficients of the original and shifted unit cells.

\section{Conclusions}

Moving reference planes of nonreciprocal unit cells of NRLSPTLSs are analyzed in this paper using the equivalent BCITL model. In the analysis, both standard and proposed formulas are used. It is found that both approaches provide identical results. However, the proposed formulas provide more insight about the relationship between associated BCITL parameters of the original and shifted unit cells. Interestingly, the equivalent BCITL characteristic impedances of the original and shifted unit cells are mathematically related by the bilinear transformation, as well as the associated load reflection coefficients of the original and shifted unit cells. In addition, the equivalent BCITL complex propagation constants remain unchanged for both unit cells as expected.

\section{Acknowledgments}

This work has been supported by the Thailand Research Fund under the TRF Senior Research Scholar Program with the contract number RTA5780010.

\section{References}

[1] POZAR, D. M. Microwave Engineering. $2^{\text {nd }}$ ed., John Wiley \& Sons, 1998. ISBN:9780471170969

[2] CALOZ, C., ITOH, T. Electromagnetic Metamaterials Transmission Line and Theory and Microwave Applications. Wiley-IEEE Press, 2005. ISBN: 9780471669852

[3] LEE, M., KRAMER, B. A., CHEN, C., et al. Distributed lumped loads and lossy transmission line model for wideband spiral antenna miniaturization and characterization. IEEE Transactions on Antennas and Propagation, 2007, vol. 55, no. 10, p. 1671 to 1678. ISSN: 0018-926X. DOI: 10.1109/TAP.2007.905823

[4] YANG, B., SKAFIDAS, E., EVANS, R. J. Slow-wave slot microstrip transmission line and bandpass filter for compact millimetre-wave integrated circuits on bulk complementary metal oxide semiconductor. IET Transaction on Microwaves, Antennas \& Propagation, 2012, vol. 6, no. 14, p. 1548-1555. ISSN: 17518725. DOI: 10.1049 /iet-map.2012.0336

[5] COLLIN, R. E. Foundations for Microwave Engineering. 2nd ed. Hoboken (NJ): Wiley/IEEE, 2001. ISBN: 0780360311

[6] SPAULDING, W. G. The application of periodic loading to a ferrite phase shifter design. IEEE Transactions on Microwave Theory and Techniques, 1971, vol. 19, no. 12, p. 922-928. DOI: 10.1109/TMTT.1971.6373342

[7] KHARADLY, M. M. Z. Periodically loaded nonreciprocal transmission lines for phase-shifter applications. IEEE Transactions on Microwave Theory and Techniques, 1974, vol. 22, no. 6, p. 635-640. DOI: 10.1109/TMTT.1974.1128305

[8] ENEGREN; T. A., KHARADLY, M. M. Z. An investigation of nonreciprocal periodic structures. IEEE Transactions on Microwave Theory and Techniques. 1980, vol. 28, no. 8, p. 905-914. DOI: 10.1109/TMTT.1980.1130190

[9] ENEGREN, T. A., KHARADLY, M. M. Z. Higher order mode interaction in nonreciprocal periodic structures. IEEE Transactions on Microwave Theory and Techniques, 1982, vol. 30, no. 5, p. 809-812. DOI: 10.1109/TMTT.1982.1131142

[10] THEOFANOPOUlOS, P. C., LAVRANOS, C. S., ZOIROS, K., et al. FDFD eigenanalysis of non-reciprocal periodic structures. In Antennas \& Propagation Conference (LAPC). Loughborough (UK), 2015, p. 1-5. DOI: 10.1109/LAPC.2015.7366019

[11] LAMULTREE, S., TORRUNGRUENG, D., AKKARAEKTHALIN, P. Analysis of reciprocal lossy periodic transmission-line structures using bi-characteristic-impedance transmission lines and Meta-Smith charts. In Proceedings of the 2015 12th International Conference on Electrical Engineering/Electronics, Computer, Telecommunications and Information Technology. Hua-Hin (Thailand), 2015. DOI: 10.1109/ECTICon.2015.7207026

[12] PISSOORT, D., OLYSLAGER, F. Study of eigenmodes in periodic waveguides using the Lorentz reciprocity theorem. IEEE Transactions on Microwave Theory and Techniques, 2004, vol. 52, no. 2, p. 542-553. DOI: 10.1109/TMTT.2003.821906

[13] YAGHJIAN, A. D. Bidirectionality of reciprocal, lossy or lossless, uniform or periodic waveguides. IEEE Microwave and Wireless Components Letters, 2007, vol. 17, no. 7, p. 480-482. DOI: 10.1109/LMWC.2007.899294

[14] LERTSIRIMIT, C., TORRUNGRUENG, D. Analysis of active loaded transmission line using an equivalent BCITL model. In Proceeding of the 2007 Asian-Pacific Microwave Conference. Bangkok (Thailand). 2007, vol. 4, p. 2353-2356.

[15] ABLOWITZ, M. J., FOKAS, A. S. Complex Variables. New York: Cambridge University Press, 2003. ISBN: 9780521534291

[16] SILAPUNT, R., TORRUNGRUENG, D. Theoretical study of microwave transistor amplifier design in the conjugately characteristic-impedance transmission line (CCITL) system using a bilinear transformation approach. Progress in Electromagnetics Research, 2011, vol. 120, p. 309-326. DOI:10.2528/PIER11080504

\section{About the Authors ...}

Suthasinee LAMULTREE (corresponding author) was born in Thailand. She received the B.Eng and M.Eng in 
Telecommunication Engineering from King Mongkut's Institute of Technology Ladkrabang, Thailand, in 2000 and 2003, respectively. In 2009, she received D.Eng in Electrical Engineering from the same institute. She joined the Electrical and Electronic Engineering Department in the Faculty of Engineering and Technology at Asian University, Thailand, in 2006. In 2016, she moved to join the Department of Electronics and Telecommunication Engineering, Faculty of Engineering, Rajamangala University of Technology Isan Khonkaen Campus, Khonkaen, Thailand. Her research interests are antennas, electromagnetics and microwave engineering.

Prayoot AKKARAEKTHALIN received the B.Eng. and M.Eng. degrees in Electrical Engineering from King Mongkut's University of Technology North Bangkok, Thailand, in 1986 and 1990, respectively, and the Ph.D. degree from the University of Delaware, Newark, USA, in 1998. From 1986 to 1988 , he worked in the Microtek Laboratory, Thailand. In 1988, he joined the Department of Electrical Engineering, KMUTNB. His current research interests include passive and active microwave circuits, wideband and multiband antennas, and telecommunication systems. He was the Chairman for the IEEE MTT/AP/ED Thailand Joint Chapter during 2007 and 2008, and the president for ECTI Association, Thailand from 2014 to 2015. He is now working on the Senior Research Scholar Project for Thailand Research Fund (2015-2017).

Danai TORRUNGRUENG received his B.Eng. degree in Electrical Engineering from Chulalongkorn University, Bangkok, Thailand, in 1993. He obtained his M.S. and Ph.D. degrees in Electrical Engineering from The Ohio State University in 1996 and 2000, respectively. Prior to joining Asian University, he worked as a senior engineer in the USA, involved in research and development of the urban propagation modeling project. At present, he is a full professor in the Electrical and Electronic Engineering Department in the Faculty of Engineering and Technology at Asian University, Thailand. His research interests are in the areas of fast computational electromagnetics, rough surface scattering, propagation modeling, electromagnetic wave theory, microwave theory and techniques, antennas and sensors. 